\title{
Negative differential resistance and electroluminescence from InAs light-emitting diodes grown by liquid-phase epitaxy
}

\author{
A. Krier and X. L. Huang \\ Department of Physics, Lancaster University, Lancaster, LA1 4YB, United Kingdom
}

(Received 20 September 2004; accepted 14 December 2004; published online 3 February 2005)

\begin{abstract}
Negative differential resistance has been observed from InAs homojunction light-emitting diodes grown using liquid-phase epitaxy at $455^{\circ} \mathrm{C}$. The devices were characterized using current-voltage $(I-V)$ and electroluminescence spectroscopy measurements to obtain information about structure defects in InAs. Two distinct negative differential resistance regions were observed in the forward bias $I-V$ characteristic, consistent with carriers tunnelling into defect levels within the InAs band gap. At large forward bias, carrier injection into the defect levels resulted in electroluminescence peaking at $372 \mathrm{meV}$ and then at $392 \mathrm{meV}$ with increasing current. Analysis based on a native lattice complex defect indicates that carriers recombine via the defect levels at temperatures up to 175 K. (C) 2005 American Institute of Physics. [DOI: 10.1063/1.1863446]
\end{abstract}

InAs and its alloys are promising materials for the fabrication of mid-infrared optoelectronic devices for use in gas sensors and many other applications. ${ }^{1}$ The nature and concentration of structure defects and their corresponding activation energies present in InAs depends on the different growth methods and conditions employed. This in turn affects the optoelectronic properties of the material and devices. As shown in Fig. 1, three deep levels $\left(E_{D}, E_{A 1}\right.$, and $E_{A 2}$ ) located at $20 \mathrm{meV}$ below the conduction band $E_{C}, 14$ and $35 \mathrm{meV}$ above the valence band $E_{V}$, respectively, are associated with the most commonly observed transitions in low temperature photoluminescence (PL) investigations of InAs. ${ }^{2,3}$ Furthermore, it is known that relatively high densities of structure defects and deep states arise in samples grown using liquid phase epitaxy (LPE) at temperatures below $550{ }^{\circ} \mathrm{C}$. ${ }^{1}$ In this paper we report unusual negative differential resistance (NDR) effects which involve the defect levels within the band gap of the active region in InAs lightemitting diodes (LEDs) grown at low temperature and provide evidence for the formation of a complex defect.

The InAs $p-i$ - $n$ LEDs were produced by LPE using a low growth temperature of $455^{\circ} \mathrm{C}$. The samples were grown from In-rich solution on the $p$-type InAs (100) substrate which was $\mathrm{Zn}$-doped to $1 \times 10^{18} \mathrm{~cm}^{-3}$. One sample containing quantum dots (QDs) was grown at $455^{\circ} \mathrm{C}$ with $0.4^{\circ} \mathrm{C} / \mathrm{min}$ ramping rate and layer contact times $\operatorname{InAs}(20 \mathrm{~s}) / \operatorname{InSb}(1 \mathrm{~ms}) / \operatorname{InAs}(10 \mathrm{~s}) / n^{+}-\operatorname{InAs}(10 \mathrm{~s})$. All the three InAs layers were grown using $30{ }^{\circ} \mathrm{C}$ supercooling, and only the $n^{+}$-InAs layer was intentionally doped with Te to $1 \times 10^{18} \mathrm{~cm}^{-3}$. The InSb QD layer was grown using $20{ }^{\circ} \mathrm{C}$ supercooling. The total thickness of the epilayers was approximately $4 \mu \mathrm{m}$. The resulting samples were fabricated into $380 \mu \mathrm{m}$ diameter mesa etched LEDs using conventional photolithography and metallization techniques. The LED chips were mounted on to TO-49 diode headers and were characterised using a combination of constant and pulsed current-voltage $(I-V)$ and electroluminescence (EL) measurements. Two types of LEDs were produced: (i) conventional LEDs containing unintentionally doped InAs and (ii) with a single layer of InSb quantum dots (QD) in the active region, introduced using a rapid slider LPE technique where the growth solution is quickly wiped across the substrate. (In the present case the QD layer was grown using a contact time of $1 \mathrm{~ms}$ at $455^{\circ} \mathrm{C}$ ). The quantum dots obtained in this way were $5 \mathrm{~nm}$ in height and $20 \mathrm{~nm}$ in diameter with an area density of $\sim 10^{10} \mathrm{~cm}^{-2}$.

The $I-V$ test set-up, is shown in Fig. 1. A computer controlled $I-V$ measurement system was used based on a National Semiconductor LM759CP power operational amplifier as a voltage source. Because of the negative resistance obtained in our devices, in order to excite electroluminescence, a supplementary circuit was used to provide dc bias to the sample as shown in Fig. 1. The pulse current amplitude (PCA, 50\% duty cycle) was measured with a Hitachi V252 oscilloscope. The low temperature EL spectra were investi-

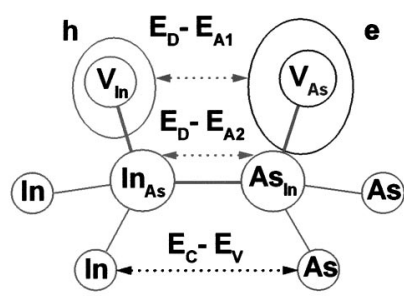

(a)

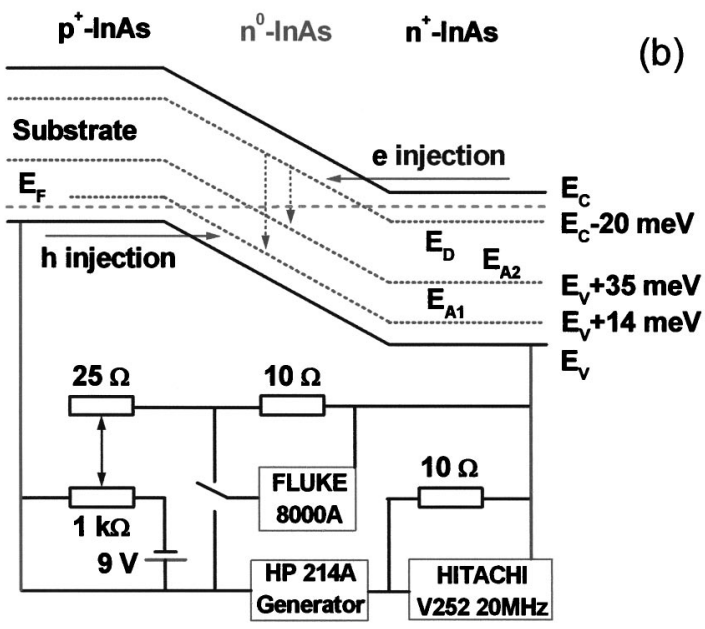

FIG. 1. (a) Schematic diagram of a structure defect complex composed of an antisite pair $\operatorname{In}_{\mathrm{As}}-\mathrm{As}_{\mathrm{In}}$ and a vacancy pair $V_{\mathrm{In}}-V_{\mathrm{As}} ;$ (b) the energy band structure and the test circuit used for the $I-V$ characterization. 


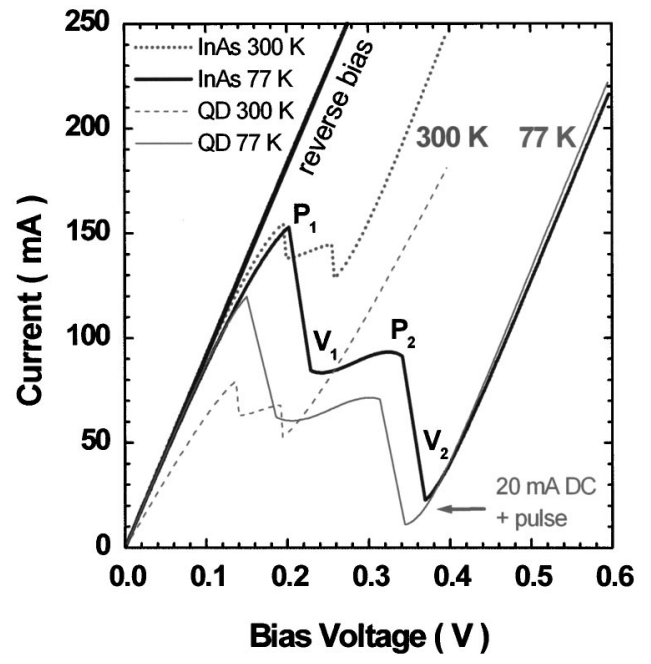

FIG. 2. $I-V$ characteristics of the InAs and QD LEDs at 77 and $300 \mathrm{~K}$. The forward bias $I-V$ of the LEDs exhibits two negative differential resistance regions with similar current drop. The reverse bias $I-V$ of the InAs LED at $77 \mathrm{~K}$ is shown for comparison.

gated by bonding the LEDs to the cold finger of an Oxford Instruments continuous flow liquid helium cryostat. The EL was dispersed by a Bentham M300 Monochromator, and detected using a liquid-nitrogen-cooled $\mathrm{InSb}$ detector and a Stanford SR830 lock-in amplifier.

With reference to Fig. 1, it has been proposed that the donor level $E_{D}$ in $n$-type InAs is related to the As vacancy $\left(V_{\mathrm{As}}\right)$ structure defect. ${ }^{5}$ The acceptor levels $E_{A 1}$ and $E_{A 2}$ originate from the doubly charged structure defect $\left(V_{\text {In }}\right.$ $\left.-\mathrm{In}_{\mathrm{As}}\right)$, a complex of an In vacancy $\left(V_{\mathrm{In}}\right)$ with an In antisite defect $\left(\operatorname{In}_{\mathrm{As}}\right)$, which tends to form in $p$-type InAs. ${ }^{6,7}$ In a perfect InAs lattice, an In atom is bonded to four As atoms and vice versa. However, when an In atom exchanges position with a neighboring As atom, an antisite pair $\mathrm{In}_{\mathrm{As}}-\mathrm{As}_{\mathrm{In}}$ is formed. The $\mathrm{In}$ atom on the antisite $\mathrm{In}_{\mathrm{As}}$ is then bonded to three In atoms and one As atom on the antisite $\mathrm{As}_{\mathrm{In}}$, which should generate an In vacancy $\left(V_{\text {In }}\right)$ to minimize energy (and vice-versa). Therefore, we suppose that it is possible to form a structure defect complex composed of an antisite pair $\mathrm{In}_{\mathrm{As}}-\mathrm{As}_{\text {In }}$ and a vacancy pair $V_{\text {In }}-V_{\mathrm{As}}$ [as shown in Fig. 1(a)]. The As vacancy $\left(V_{\mathrm{As}}\right)$ may attract an electron and interact with an As antisite $\left(\mathrm{As}_{\text {In }}\right)$ to form a deep donor state $E_{D}$. The In vacancy $\left(V_{\text {In }}\right)$ may attract a hole but is less likely to interact with an In antisite $\left(\operatorname{In}_{\mathrm{As}}\right)$ due to the more localized wave function of the hole, thus it forms two deep acceptor states $E_{A 1}$ and $E_{A 2}$ instead.

Figure 2 clearly shows the negative differential resistance (NDR) effect, which is associated with tunnelling, observed in the $I-V$ characteristics measured from each of the LEDs. At $77 \mathrm{~K}$ the forward bias current of the InAs LED increases sublinearly with increasing bias voltage to $P_{1}(0.2020 \mathrm{~V}, 152.9 \mathrm{~mA})$ and drops to $V_{1}(0.2287 \mathrm{~V}$, $84.6 \mathrm{~mA})$, then slowly increases to $P_{2}(0.3417 \mathrm{~V}, 91.0 \mathrm{~mA})$, drops to $V_{2}(0.3693 \mathrm{~V}, 22.8 \mathrm{~mA})$, and then increases again almost linearly with increasing bias voltage. The NDR region from $P_{1}$ to $V_{1}(68.3 \mathrm{~mA})$ is almost equal to that from $P_{2}$ to $V_{2}(68.2 \mathrm{~mA})$, but in a different current range. This implies that the NDR may involve a similar number of carriers or deep level states in two parallel channels ${ }^{8}$ and that the corresponding donor and acceptor states $E_{D}-E_{A 1}$ and

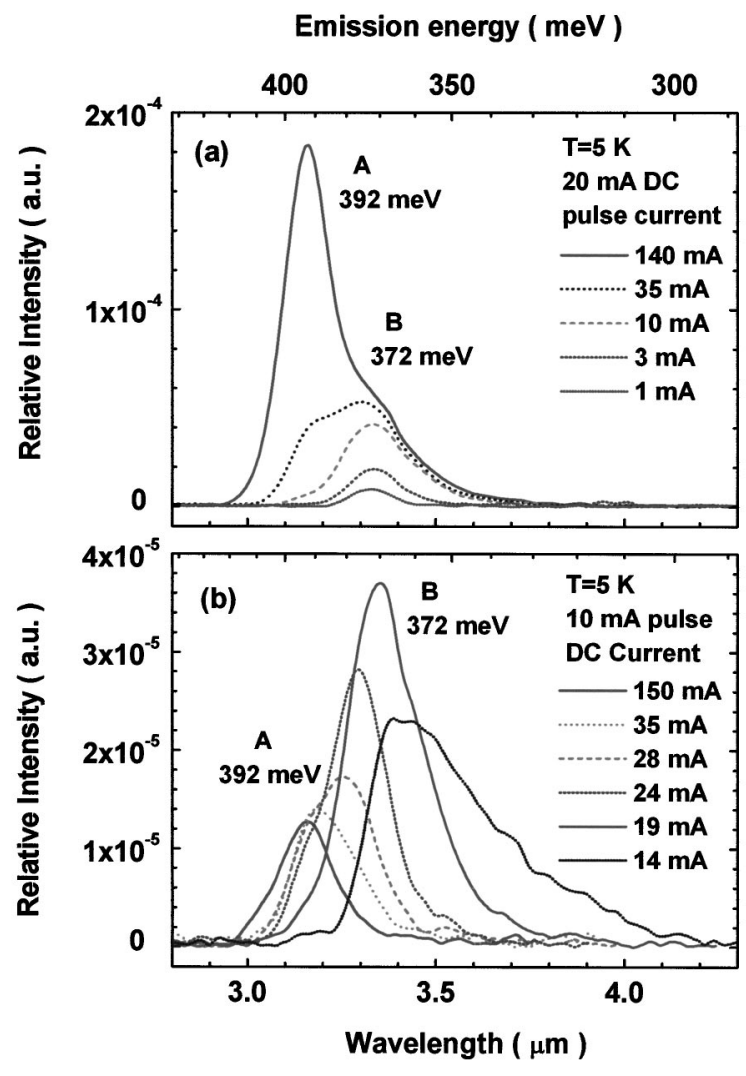

FIG. 3. Current dependence of the EL spectrum of the InAs QD LED at $5 \mathrm{~K}$. (a) The LED was firstly biased to $0.37 \mathrm{~V}$ at $20 \mathrm{~mA} \mathrm{dc}$, and then different pulse currents (PCA) were superimposed. (b) The LED operated using a fixed pulse current amplitude (PCA) of $10 \mathrm{~mA}$ with different dc currents superimposed at large bias voltage $(>0.35 \mathrm{~V})$.

$E_{D}-E_{A 2}$ could be associated in pairs within a complex.

Electron tunnelling takes place from the $n^{+}$-InAs layer to the donor levels at $E_{D}\left(20 \mathrm{meV}\right.$ below $\left.E_{c}\right)$ and reaches a peak at $P_{1}$. The voltage at $P_{1}(152.9 \mathrm{~mA})$ is $36 \mathrm{mV}$ higher than that in reverse bias at the same current. This means the voltage drop across the undoped InAs active region in forward bias is $\sim 36 \mathrm{mV}$. The decrease in current from $P_{1}$ to $V_{1}$ relates to the off-resonant tunnelling into the donor states, and similarly the decrease from $P_{2}$ to $V_{2}$ relates to offresonant tunnelling into the acceptor states, since $E_{V}-E_{A 2}$ (35 meV) is roughly twice as large as $E_{C}-E_{D}(20 \mathrm{meV})$ consistent with Fig. 1.

At $300 \mathrm{~K}$, the InAs LED (without QD in the active region) again shows a double NDR, but the total current only decreased by $16 \%$ from $P_{1}$ to $V_{2}$, which is much less than at $77 \mathrm{~K}(85 \%)$. At $300 \mathrm{~K}$ most of the donor-acceptor pairs in the undoped InAs active layer are dissociated, the electrons and holes become thermally reactivated back into the respective energy bands and are not available for tunnelling.

At $77 \mathrm{~K}$, the $I-V$ peaks and valleys of the InSb QD LED are $\quad P_{1}(0.1502 \mathrm{~V}, 119.9 \mathrm{~mA}), \quad V_{1}(0.1857 \mathrm{~V}, 62.4 \mathrm{~mA})$, $P_{2}(0.3138 \mathrm{~V}, 70.8 \mathrm{~mA})$, and $V_{2}(0.3444 \mathrm{~V}, 11.3 \mathrm{~mA})$, respectively. The current drop from $P_{1}$ to $V_{1}$ is again similar to that from $P_{2}$ to $V_{2}$, and the total current reduced by $90 \%$ from $P_{1}$ to $V_{2}$. At $300 \mathrm{~K}$, in comparison with the values measured at $77 \mathrm{~K}$, the current at $P_{1}$ is significantly reduced $(34 \%)$ as is the peak voltage. This is a direct consequence of the presence of InSb QDs which have the effect of generating many additional local defects and corresponding strainAIP license or copyright; see http://apl.aip.org/apl/copyright.jsp 


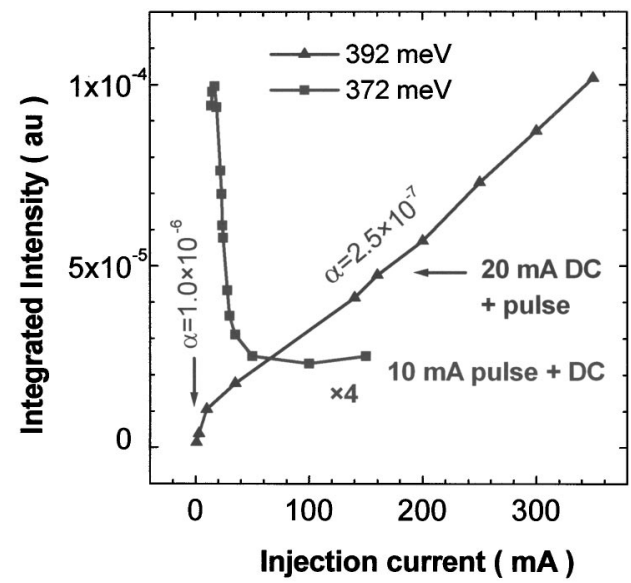

FIG. 4. The dc current and PCA dependencies of the integrated EL intensity of the InSb QD LED at $5 \mathrm{~K}$.

induced donor-acceptor pairs in the undoped InAs active layer. Although emission is obtained from these LEDs, no EL emission is observed from the QD themselves in these devices.

In order to observe light emission, the QD LED was firstly biased to $0.37 \mathrm{~V}$ at $20 \mathrm{~mA} \mathrm{dc}$, and then different pulse currents were superimposed. The resulting dependence of the $\mathrm{EL}$ at $5 \mathrm{~K}$ is shown in Fig. 3(a). At small PCA $(<10 \mathrm{~mA})$ only peak B was observed, centered at $372 \mathrm{meV}$, corresponding to the transition from $E_{D}$ to $E_{A 2}$. However, due to carrier filling effects at large PCA, peak B became saturated and peak A appeared centred at $392 \mathrm{meV}$, corresponding to the transition from $E_{D}$ to $E_{A 1}$. We estimated that $E_{A 2}$ was fully filled at about $30 \mathrm{~mA}$ PCA $+20 \mathrm{~mA}$ dc (which is approximately equivalent to $35 \mathrm{~mA}$ dc injection). Figure 3(b) shows the dependence of the EL spectra on dc injection at a fixed PCA of $10 \mathrm{~mA}$. Peak B reached a maximum EL intensity at $19 \mathrm{~mA}$ dc injection with a transition energy of $372 \mathrm{meV}$ before peak A began to appear. The switching behavior of these two peaks with injection current suggests that the corresponding radiative transitions are associated with carriers recombining together within complexes containing the respective structure defects associated with appropriate donor and acceptor levels.

As shown in Fig. 4, the integrated EL intensity increases almost linearly in both small PCA $(0-10 \mathrm{~mA})$ and large PCA (10-350 mA) regions using $20 \mathrm{~mA}$ dc injection, but the slope at small PCA related to the $372 \mathrm{meV}$ peak (B) is about 4 times that observed using large PCA dominated by the $392 \mathrm{meV}$ peak (A). On the other hand at fixed $10 \mathrm{~mA}$
PCA, the integrated EL intensity at small dc current $(14-19 \mathrm{~mA})$ is about 4 times that observed at large dc current (50-150 mA). Since the slope represents the LED differential efficiency this suggests that the radiative efficiency of the $372 \mathrm{meV}$ peak is about 4 times as high as that of the $392 \mathrm{meV}$ peak due to stronger confinement for the lower energy transition in the complex. We suppose that the electron and hole injected into $V_{\mathrm{As}}$ and $V_{\mathrm{In}}$ are strongly confined by the complex, and the corresponding donor-acceptor pair $E_{D}-E_{A 2}$ has stronger confinement than that of $E_{D}-E_{A 1}$. Therefore, the EL peak B exhibits a higher radiation efficiency (4 times that of A), as well as intensity saturation at large injection. Furthermore, it is also insensitive to temperature in peak position and intensity up to $175 \mathrm{~K}$. This is consistent with the characteristics of radiative recombination originating from a complex which gives out intense luminescence that quenches slowly and persists up to high temperature.

In conclusion, negative differential resistance and electroluminescence have been observed from InAs LEDs containing InSb quantum dots grown by LPE at $455^{\circ} \mathrm{C}$. A complex structure defect composed of an antisite pair $\operatorname{In}_{\mathrm{As}}-\mathrm{As}_{\text {In }}$ and a vacancy pair $V_{\text {In }}-V_{\text {As }}$ has been suggested to account for the experimental observations. The interaction of $V_{\mathrm{As}}$ and $A s_{\text {In }}$ results in the formation of a deep donor state $E_{D}$, while the interaction of $V_{\text {In }}$ and $\operatorname{In}_{\mathrm{As}}$ generates two deep acceptor states $E_{A 1}$ and $E_{A 2}$. Carriers may resonantly tunnel into the deep states at small bias, which become filled by carrier injection at large forward bias. At low temperature the EL efficiency of the $372 \mathrm{meV}$ peak is about 4 times that of the $392 \mathrm{meV}$ peak. The electron-hole pair confined by the complex was observed to exhibit intense EL up to about $175 \mathrm{~K}$.

The authors wish to thank EPSRC for the award of a research grant to support this work.

${ }^{1}$ M. Fisher and A. Krier, Infrared Phys. Technol. 38, 405 (1997).

${ }^{2}$ P. J. P. Tang, C. C. Phillips, and R. A. Stradling, Semicond. Sci. Technol. 8, 2135 (1993).

${ }^{3}$ H. H. Gao, A. Krier, and V. V. Sherstnev, Semicond. Sci. Technol. 14, 441 (1999).

${ }^{4}$ A. Krier, X. L. Huang, and A. Hammiche, J. Phys. D 34, 874 (2001).

${ }^{5}$ A. N. Baranov, T. I. Voronina, A. A. Gorelenok, T. S. Lagunova, A. M. Litvak, M. A. Sipovskaya, S. P. Starosel'tseva, V. V. Sherstnev, and Yu. P. Yakovlev, Sov. Phys. Semicond. 26, 905 (1992).

${ }^{6}$ A. N. Baranov, T. I. Voronina, T. S. Lagunova, M. A. Sipovskaya, V. V. Sherstnev, and Yu. P. Yakovlev, Semiconductors 27, 236 (1993).

${ }^{7}$ T. I. Voronina, B. E. Dzhurtanov, T. S. Lagunova, M. A. Sipovskaya, V. V. Sherstnev, and Yu. P. Yakovlev, Semiconductors 32, 250 (1998).

${ }^{8}$ U. M. Khan-Cheema, P. C. Klipstein, D. G. Austing, J. M. Smith, N. J. Mason, P. J. Walker, and G. Hill, Solid-State Electron. 37, 977 (1994). 